\title{
Momentum Dumping for Space Robots
}

\author{
Alessandro M. Giordano ${ }^{a, b}$, Gianluca Garofalo $^{a}$, Alin Albu-Schäffer ${ }^{a, b}$
}

\begin{abstract}
During the robotic capture of a target object on orbit, accidental contacts may happen. During contacts, momentum is transferred to the system, causing a drift of the space robot in the inertial space. When no remediation is taken, the arm might converge to singularity or workspace limit within seconds, compromising the capture operation. This article presents a method to control the end-effector while simultaneously extracting any accumulated momentum in the system to cancel the drift. A feature of the method is that external actuators are only used for the momentum extraction and not to counterbalance the manipulator control forces. The control is validated with experiments using a Hardware-In-theLoop (HIL) robotic simulator composed of a 7DOF (Degrees Of Freedom) arm mounted on a 6DOF moving base.
\end{abstract}

\section{INTRODUCTION}

The future use of space robots will require efficient coordination of the manipulator and satellite motion. In a space robot, the coupled dynamic behavior between the arm and the satellite on which the manipulator is mounted imposes great challenges from the point of view of the control of the motion. Several control strategies have been investigated in the past years to tackle this problem. They can be mainly divided in two categories: the free-floating strategies, in which the satellite actuators are turned off to save fuel, and the actuated-base strategies, in which the actuators are used to accomplish additional tasks. In the first category, the dynamics can be reduced using the conservation of momentum [1]. Based on this, while the end effector is commanded to reach a desired location, the base is left free to move. While the free-floating techniques avoid the use of satellite thrusters, saving precious fuel, some drawbacks limit their applicability to full on-orbit operations. When linear and angular momenta are nonzero, e.g. after contacts or under the effect of orbital disturbances, the space robot drifts in the inertial system [2]. Although free-floating controllers have been proposed for those situations [3], [4], [5], the endeffector convergence in the inertial space can be ensured only for a limited time, before reaching a singularity or the workspace limit [6]. This situation is exemplified in Fig 1 In [6], the external actuators are used to remove the linear momentum drift, fixing the relative end-effector/base position. However, the effects of the angular momentum drift are not taken into account and this may lead to reaching workspace limits or singularity. Additionally, trying to rigidly control the base position [6], or even the orientation [7] could lead to excessive thrusters usage. In fact, the task of rigidly

\footnotetext{
${ }^{a}$ The authors are with the Institute of Robotics and Mechatronics, German Aerospace Center (DLR), 82234 Weßling, Germany

${ }^{b}$ The authors are with the Department of Informatics, Technical University of Munich (TUM), Garching, 85748, Germany
}

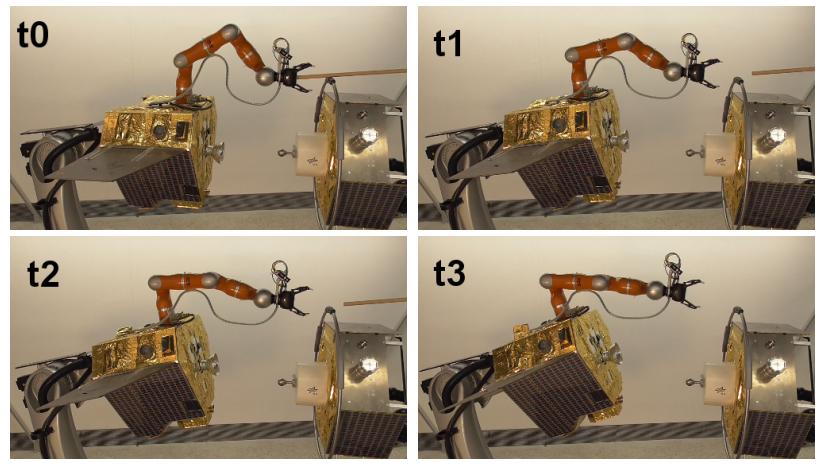

Fig. 1. Snapshot sequence of a free-floating robot in response to an accidental contact. The satellite starts to drift, the robot reaches a singular configuration and the capture operation is compromised.

fixing the base alters considerably the natural floating-base behavior of the system and may be too demanding for the satellite actuators, leading to saturation and/or excessive fuel consumption. When only the drift removal is of interest, a better solution would be to leave the base floating and use the thrusters only to extract the accumulated momentum from the system. With this approach, the system could be brought to rest by applying only the minimal amount of satellite actuation corresponding to the accumulated momentum.

In this paper, a method is proposed to control the end effector in the inertial frame while simultaneously extracting any accumulated momentum in the system. A feature of the method is that external actuators are only used for the momentum extraction and not to counterbalance the manipulator control forces. For this purpose, the idea already present in the literature of an internal/external motion decomposition is further developed to systematically assign the end effector task only to internal forces. The control is validated with experiments using a HIL facility consisting of a redundant torque-controlled robot mounted on a full 3D satellite robotic simulator. The main contributions of the paper are:

1) The derivation of an internal/external force decomposition for the end effector.

2) The derivation of a momentum dumping ${ }^{1}$ control as an alternative fuel efficient strategy for the control of space robots subject to external interactions.

3) Validating, to the best of our knowledge for the first time, a space robot controller using a torque-controlled robot and a full 3D robotic simulator.

\footnotetext{
${ }^{1}$ In the astronautical literature, the term "dumping" refers to the extraction of the momentum accumulated in momentum storage devices. Here it is more generally used to refer to the extraction of momentum from the whole multibody system, i.e. also including the arm.
} 
The paper is organized as follows. In Sect. II we preliminary introduce the scenario and the main dynamics formulation. In Sect. III the external/internal motion decomposition is addressed. In Sect. IV the momentum dumping control is derived. Sect. V presents the experimental results. Finally, the conclusions are given in Sect. VI.

\section{Preliminaries}

\section{A. Problem statement}

A serial-link space robot composed of $n+1$ bodies is considered, where $n$ is the number of joints of the arm. The satellite base is fully actuated in translation and rotation by means of an external generalized force $\boldsymbol{f}_{b} \in \mathbb{R}^{6}$. No environmental forces are considered during the motion, since they are orders of magnitude less than the driving torques $\tau \in \mathbb{R}^{n}$ acting at the manipulator joints.

The operational scenario is that of capturing a target object in presence of initial momenta. This may be the post-contact situation after an accidental impact with the target or a nominal capture situation under the accumulated effect of orbital disturbances. Assuming the target object is stationary in the inertial space, the robot end-effector is commanded to reach a desired position and orientation in the inertial space.

\section{B. Space robot dynamics and main notation}

We call $\mathcal{B}$ the body frame of the satellite around its CoM (Center of Mass), $\mathcal{E}$ the end-effector frame and $\mathcal{T}$ a target frame fixed in the inertial space which defines the desired EE (End-Effector) pose. Additionally, a frame $\mathcal{C}$ is placed on the CoM of the whole system always parallel to $\mathcal{T}$. Let us indicate with the symbol $\boldsymbol{\nu}_{x} \in \mathbb{R}^{6}$ the inertial velocity of a general body frame $\mathcal{X}$ expressed in the body frame and with the symbol $\boldsymbol{f}_{x} \in \mathbb{R}^{6}$ the generalized force acting on the body, also expressed in the body frame. The dynamics of the space robot is described by the equation [8]:

$$
\boldsymbol{M}(\boldsymbol{q}) \dot{\boldsymbol{v}}+\boldsymbol{C}(\boldsymbol{q}, \boldsymbol{v}) \boldsymbol{v}=\boldsymbol{f}
$$

The symbols used here are defined as follows: $M \in$ $\mathbb{R}^{(6+n) \times(6+n)}$ inertia matrix of the space robot; $\boldsymbol{C} \in$ $\mathbb{R}^{(6+n) \times(6+n)}$ Coriolis and centrifugal matrix; $\boldsymbol{q} \in Q$ joint angles ${ }^{2} \dot{\boldsymbol{q}} \in \mathbb{R}^{n}$ joint velocities; $\boldsymbol{v} \in \mathbb{R}^{6+n}$ generalized velocities $\left[\begin{array}{cc}\boldsymbol{\nu}_{b}^{T} & \dot{\boldsymbol{q}}^{T}\end{array}\right]^{T} ; \boldsymbol{\nu}_{b} \in \mathbb{R}^{6}$ base velocity; $\boldsymbol{f} \in \mathbb{R}^{6+n}$ generalized forces $\left[\begin{array}{ll}\boldsymbol{f}_{b}^{T} & \boldsymbol{\tau}^{T}\end{array}\right]^{T}$. Writing the symmetric inertia matrix in block diagonal form, it is

$$
\boldsymbol{M}=\left[\begin{array}{cc}
\boldsymbol{M}_{b} & \boldsymbol{M}_{b m} \\
\boldsymbol{M}_{b m}^{T} & \boldsymbol{M}_{m}
\end{array}\right],
$$

where $M_{b} \in \mathbb{R}^{6 \times 6}$ is the inertia matrix of the whole system around $\mathcal{B}, M_{b m} \in \mathbb{R}^{6 \times n}$ is the coupling submatrix and $M_{m} \in \mathbb{R}^{n \times n}$ is the inertia matrix of the manipulator.

The end-effector velocity $\boldsymbol{\nu}_{e} \in \mathbb{R}^{6}$ is given by

$$
\begin{array}{r}
\boldsymbol{\nu}_{e}=\boldsymbol{J} \boldsymbol{v}=\left[\begin{array}{ll}
\boldsymbol{J}_{b} & \boldsymbol{J}_{m}
\end{array}\right] \boldsymbol{v}, \\
{ }^{2} Q=\underbrace{\mathbb{S}^{1} \times \cdots \times \mathbb{S}^{1}}_{n} \text { and } \mathbb{S}^{1} \text { is the unit circle. }
\end{array}
$$

with $\boldsymbol{J}_{b} \in \mathbb{R}^{6 \times 6}, \boldsymbol{J}_{m} \in \mathbb{R}^{6 \times n}$. Let us now indicate with $\boldsymbol{h} \in \mathbb{R}^{6}$ the generalized momentum of the whole system, i.e. the sum of the momenta of each body, around the frame $\mathcal{C}$. The momentum can be expressed as a linear combination of the generalized velocities $\boldsymbol{v}$ through $\boldsymbol{L} \in \mathbb{R}^{6 \times(6+n)}$, as [1]

$$
\boldsymbol{h}=\boldsymbol{L} \boldsymbol{v}=\left[\begin{array}{ll}
\boldsymbol{A}^{-T} \boldsymbol{M}_{b} & \boldsymbol{A}^{-T} \boldsymbol{M}_{b m}
\end{array}\right] \boldsymbol{v}
$$

where $\boldsymbol{A}$ is the transformation matrix from the frame $\mathcal{C}$ to the frame $\mathcal{B}$, defined as

$$
\boldsymbol{A}=\left[\begin{array}{cc}
\boldsymbol{R}_{c b} & {\left[\boldsymbol{p}_{c b}\right]^{\wedge} \boldsymbol{R}_{c b}} \\
\mathbf{0} & \boldsymbol{R}_{c b}
\end{array}\right]
$$

$\boldsymbol{p}_{x y} \in \mathbb{R}^{3}$ and $\boldsymbol{R}_{x y} \in \mathbb{R}^{3 \times 3}$ generically indicate respectively the position vector and the rotation matrix from a frame $\mathcal{X}$ to a frame $\mathcal{Y}$. The operator $[\cdot]^{\wedge}$ indicates the skewsymmetric matrix of the vector argument. Defining a local set of coordinates [9] $\tilde{\boldsymbol{x}}_{e} \in \mathbb{R}^{6}$ for the position and rotation from the frame $\mathcal{T}$ to the frame $\mathcal{E}$, it is possible ${ }^{3}$ to express $\boldsymbol{A}$ as $\boldsymbol{A}\left(\boldsymbol{q}, \tilde{\boldsymbol{x}}_{e}\right)$.

In the following section it will be shown how it is possible to decompose the dynamics (1) in external and internal components. The decomposition will be addressed in the end effector space to allow the derivation of Cartesian controllers.

\section{INTERNAL AND EXTERNAL MOTION DECOMPOSITION}

The motion of a floating multibody system can be decomposed into an internal motion due to the joints and an external motion as a whole object floating in the inertial space.

In joint space, the decomposition can be obtained using the momentum (4). In this case, the kinetic energy can be written as [10]

$$
T=\underbrace{\frac{1}{2} \dot{\boldsymbol{q}}^{T} \boldsymbol{M}_{m}^{*} \dot{\boldsymbol{q}}}_{T_{i n t}}+\underbrace{\frac{1}{2} \boldsymbol{h}^{T} \boldsymbol{M}_{c}^{-1} \boldsymbol{h}}_{T_{e x t}}
$$

where $\boldsymbol{M}_{m}^{*}=\boldsymbol{M}_{m}-\boldsymbol{M}_{b m}^{T} \boldsymbol{M}_{b}^{-1} \boldsymbol{M}_{b m}$ is the reduced manipulator inertia and $\boldsymbol{M}_{c}=\boldsymbol{A}^{-T} \boldsymbol{M}_{b} \boldsymbol{A}^{-1}$ is the inertia of the whole system around $\mathcal{C}$. In the following it will be shown how a similar decoupled form of the energy can be obtained for the end effector space, i.e.

$$
T=\underbrace{\frac{1}{2} \boldsymbol{\nu}_{e, i n t}^{T} \boldsymbol{M}_{e} \boldsymbol{\nu}_{e, i n t}}_{T_{i n t}}+\underbrace{\frac{1}{2} \boldsymbol{h}^{T} \boldsymbol{M}_{c}^{-1} \boldsymbol{h}}_{T_{\text {ext }}},
$$

where $\boldsymbol{\nu}_{e, i n t}$ is an end-effector internal velocity and $\boldsymbol{M}_{e}$ is the inertia associated to the internal energy. First, a precise definition for $\boldsymbol{\nu}_{e, i n t}$ is given. Second, the proposed $\left[\begin{array}{ll}\boldsymbol{h}^{T} & \boldsymbol{\nu}_{e, i n t}^{T}\end{array}\right]^{T}$ decomposition is applied on the dynamics (1) to define an end-effector internal force $\boldsymbol{f}_{e, i n t}$, and its advantageous properties are analyzed.

\footnotetext{
${ }^{3}$ In fact, $\boldsymbol{R}_{c b}=\boldsymbol{R}_{t b}=\boldsymbol{R}_{t e}\left(\tilde{\boldsymbol{x}}_{e}\right) \boldsymbol{R}_{e b}(\boldsymbol{q})$ and $\boldsymbol{p}_{c b}=$ $-\boldsymbol{R}_{c b}\left(\boldsymbol{q}, \tilde{\boldsymbol{x}}_{e}\right) \boldsymbol{p}_{b c}(\boldsymbol{q})$.
} 


\section{A. End-effector internal velocity}

The absolute motion $\nu_{e}$ of the end-effector frame can be considered as the superposition of an internal end-effector motion $\boldsymbol{\nu}_{e, i n t}$ and an external global motion of the system due to the momentum. Based on that, when the external motion is zero, the absolute end-effector motion $\boldsymbol{\nu}_{e}$ must coincide with the internal one $\boldsymbol{\nu}_{e, i n t}$. Thus, we define $\boldsymbol{\nu}_{e, \text { int }}$ such that:

$$
\boldsymbol{h}=\mathbf{0} \quad \Longleftrightarrow \quad \boldsymbol{\nu}_{e}=\boldsymbol{\nu}_{e, \text { int }},
$$

i.e. the end-effector velocity must reduce to the internal velocity when the momentum is zero.

To find the expression of $\nu_{e, i n t}$, we rewrite $\nu_{e}$ in terms of momentum. Removing the base velocity from (3) using (4), the end effector velocity $\nu_{e}$ is rewritten as

$$
\boldsymbol{\nu}_{e}=\boldsymbol{J}_{m}^{*} \dot{\boldsymbol{q}}+\boldsymbol{J}_{b} \boldsymbol{M}_{b}^{-1} \boldsymbol{A}^{T} \boldsymbol{h},
$$

where $\boldsymbol{J}_{m}^{*}=\boldsymbol{J}_{m}-\boldsymbol{J}_{b} \boldsymbol{M}_{b}^{-1} \boldsymbol{M}_{b m} \in \mathbb{R}^{6 \times n}$ is the well-known generalized Jacobian of the end effector.

From (9) it is straightforward to see that $\boldsymbol{J}_{m}^{*} \dot{\boldsymbol{q}}$ is the internal end effector velocity in the sense of (8). On the other hand, the second term in (9) is the part of end effector motion due to the global motion of the system. Therefore, we define:

$$
\boldsymbol{\nu}_{e, i n t} \triangleq \boldsymbol{J}_{m}^{*} \dot{\boldsymbol{q}}
$$

From now on, let us consider for the sake of simplicity and without loss of generality the case of nonredundant manipulator, i.e. $n=6$. For a redundant manipulator all the results can be extended using an orthogonal nullspace decomposition, as done in [5]. When $\boldsymbol{J}_{m}^{*}$ is nonsingular, using the inverse of [10] it is easy to verify that the energy decoupling (7) holds and $\boldsymbol{M}_{e}=\left(\boldsymbol{J}_{m}^{*} \boldsymbol{M}_{m}^{-1} \boldsymbol{J}_{m}^{* T}\right)^{-1}$.

\section{B. Internal/external force decomposition}

Based on the concept of end effector internal velocity, the dynamics (1) can be decomposed into an internal and an external part. To do so, a transformation matrix $\Gamma \in$ $\mathbb{R}^{12 \times(6+n)}$ is defined as

$$
\left[\begin{array}{c}
\boldsymbol{h} \\
\boldsymbol{\nu}_{e, \text { int }}
\end{array}\right]=\underbrace{\left[\begin{array}{cc}
\boldsymbol{A}^{-T} \boldsymbol{M}_{b} & \boldsymbol{A}^{-T} \boldsymbol{M}_{b m} \\
\mathbf{0} & \boldsymbol{J}_{m}^{*}
\end{array}\right]}_{\boldsymbol{\Gamma}}\left[\begin{array}{c}
\boldsymbol{\nu}_{b} \\
\dot{\boldsymbol{q}}
\end{array}\right],
$$

where (4) and (10) have been used. The generalized forces $f$ transform as

$$
\left[\begin{array}{c}
f_{b} \\
\boldsymbol{\tau}
\end{array}\right]=\Gamma^{T}\left[\begin{array}{c}
a_{h} \\
f_{e, i n t}
\end{array}\right]
$$

where $\boldsymbol{a}_{h}, \boldsymbol{f}_{e, i n t} \in \mathbb{R}^{6}$ are the new control inputs, dual respectively to $\boldsymbol{h}$ and $\boldsymbol{\nu}_{e, \text { int }}$. The equation of motion (1) can be transformed to the new state using a congruent coordinate transformation [11]. Assuming a nonsingular $\boldsymbol{J}_{m}^{*}$ it is possible to invert (11) and transform (1) as:

$$
\left[\begin{array}{cc}
\boldsymbol{M}_{c}^{-1} & \mathbf{0} \\
\mathbf{0} & \boldsymbol{M}_{e}
\end{array}\right]\left[\begin{array}{c}
\dot{\boldsymbol{h}} \\
\dot{\boldsymbol{\nu}}_{e, i n t}
\end{array}\right]+\left[\begin{array}{cc}
\boldsymbol{C}_{h} & -\boldsymbol{C}_{e h}^{T} \\
\boldsymbol{C}_{e h} & \boldsymbol{C}_{e}
\end{array}\right]\left[\begin{array}{c}
\boldsymbol{h} \\
\boldsymbol{\nu}_{e, i n t}
\end{array}\right]=\left[\begin{array}{c}
\boldsymbol{a}_{h} \\
\boldsymbol{f}_{e, \text { int }}
\end{array}\right]
$$

A first observation is that in (13) the momentum $\boldsymbol{h}$ is inertially decoupled from the end effector motion, as a consequence of the energy decoupling (7).

Secondly, the usual skew-symmetry property holds 4

$$
\boldsymbol{\nu}_{e, \text { int }}^{T}\left(\dot{\boldsymbol{M}}_{e}-2 \boldsymbol{C}_{e}\right) \boldsymbol{\nu}_{e, i n t}=\mathbf{0}, \quad \forall \boldsymbol{\nu}_{e, i n t} \in \mathbb{R}^{6} .
$$

The equation (13) can be further simplified considering that the Coriolis/centrifugal acceleration (see the Appendix) can be shown to be identically zero for the momentum, obtaining

$$
\begin{aligned}
& \boldsymbol{M}_{c}^{-1} \dot{\boldsymbol{h}}=\boldsymbol{a}_{h}, \\
& \boldsymbol{M}_{e} \dot{\boldsymbol{\nu}}_{e, i n t}+\boldsymbol{C}_{e} \boldsymbol{\nu}_{e, i n t}+\boldsymbol{C}_{e h} \boldsymbol{h}=\boldsymbol{f}_{e, \text { int }} .
\end{aligned}
$$

Notice that in the form (15), the momentum is totally decoupled from the internal end-effector motion. This leads to the main result of the proposed decomposition: by exploiting this decoupled structure is possible to design controllers in a cascade fashion, using $\boldsymbol{a}_{h}$ for the stabilization of the external motion (15a) and $\boldsymbol{f}_{e, i n t}$ to design the control of the end effector. The advantage of doing so is that, the commanded action to the thrusters will not be affected by the input $\boldsymbol{f}_{e, \text { int }}$ used to stabilize the end-effector task. This can be seen from the triangular structure of the actuation matrix (12), which can be written explicitly as

$$
\left[\begin{array}{c}
\boldsymbol{f}_{b} \\
\boldsymbol{\tau}
\end{array}\right]=\left[\begin{array}{cc}
\boldsymbol{M}_{b} \boldsymbol{A}^{-1} & \mathbf{0} \\
\boldsymbol{M}_{b m}^{T} \boldsymbol{A}^{-1} & \boldsymbol{J}_{m}^{* T}
\end{array}\right]\left[\begin{array}{c}
\boldsymbol{a}_{h} \\
\boldsymbol{f}_{e, \text { int }}
\end{array}\right] .
$$

This triangular structure is specific of the proposed force decomposition and does not hold when the absolute force $\boldsymbol{f}_{e}$ dual to $\boldsymbol{\nu}_{e}$, is used instead of $\boldsymbol{f}_{e, i n t}$.

The details of this control concept will be shown in the next section, in which the decoupled structure of the dynamics 15] is exploited to design the end effector control of a space robot with simultaneous dumping of the accumulated momentum.

\section{END EFFECTOR CONTROL AND MOMENTUM DUMPING}

The control task is to command the end effector frame $\mathcal{E}$ to a desired pose $\mathcal{T}$ in the inertial space. We require the task be accomplished only by internal actuators. Therefore, the end effector controller is defined as:

$$
\boldsymbol{f}_{e, i n t}=-\boldsymbol{J}_{\tilde{x}_{e} \nu_{e}}^{T} \boldsymbol{K}_{e} \tilde{\boldsymbol{x}}_{e}-\boldsymbol{D}_{e} \boldsymbol{\nu}_{e},
$$

where $\boldsymbol{K}_{e} \in \mathbb{R}^{6 \times 6}$ is a symmetric positive definite stiffness matrix, $\boldsymbol{D}_{e} \in \mathbb{R}^{6 \times 6}$ is a positive definite damping matrix and $\boldsymbol{J}_{\tilde{x}_{e} \nu_{e}} \in \mathbb{R}^{6 \times 6}$ is the so-called representation Jacobian of the set of coordinates $\tilde{\boldsymbol{x}}_{\boldsymbol{e}}$, which relates the time derivative of the coordinates to the end effector body velocity, as

$$
\dot{\tilde{\boldsymbol{x}}}_{e}=\boldsymbol{J}_{\tilde{x}_{e} \nu_{e}} \boldsymbol{\nu}_{e}
$$

Here, a quaternion-based representation [9] is chosen, for which

$$
\tilde{\boldsymbol{x}}_{e}=\left[\begin{array}{c}
\boldsymbol{p}_{e t} \\
2 \boldsymbol{\epsilon}
\end{array}\right] \quad \boldsymbol{J}_{\tilde{x}_{e} \nu_{e}}=\left[\begin{array}{cc}
\boldsymbol{E} & \mathbf{0} \\
\mathbf{0} & \eta \boldsymbol{E}-[\boldsymbol{\epsilon}]^{\wedge}
\end{array}\right]
$$

\footnotetext{
${ }^{4}$ The skew-simmetry holds [8] for $\boldsymbol{M}, \boldsymbol{C}$ in [1, it holds also for the transformed matrices [9, Lemma 3.2] in 13] and every submatrix.
} 
where $\eta \in \mathbb{R}, \epsilon \in \mathbb{R}^{3}$ are respectively the scalar and vector part of the quaternion extracted from $\boldsymbol{R}_{e t}$ and $\boldsymbol{E} \in \mathbb{R}^{3 \times 3}$ is the identity matrix. An additional task of momentum dumping is achieved by external actuators through

$$
\boldsymbol{a}_{h}=-\boldsymbol{M}_{c}^{-1} \boldsymbol{D}_{h} \boldsymbol{h}
$$

where $\boldsymbol{D}_{h} \in \mathbb{R}^{6 \times 6}$ is a positive definite momentum gain matrix [10].

The resulting closed-loop dynamics is obtained inserting (9), (17), (20) into (15), inserting (9), (10) into (18), and inverting $(10)$, as:

$$
\begin{aligned}
& \dot{\boldsymbol{h}}+\boldsymbol{D}_{h} \boldsymbol{h}=\mathbf{0}, \\
& \quad \boldsymbol{M}_{e} \dot{\boldsymbol{\nu}}_{e, i n t}+\boldsymbol{C}_{e} \boldsymbol{\nu}_{e, i n t}+\boldsymbol{D}_{e} \boldsymbol{\nu}_{e, i n t}+\boldsymbol{J}_{\tilde{x}_{e} \nu_{e}}^{T} \boldsymbol{K}_{e} \tilde{\boldsymbol{x}}_{e}= \\
& \quad=-\left(\boldsymbol{C}_{e h}+\boldsymbol{D}_{e} \boldsymbol{J}_{b} \boldsymbol{M}_{b}^{-1} \boldsymbol{A}^{T}\right) \boldsymbol{h}, \\
& \dot{\tilde{\boldsymbol{x}}}_{e}=\boldsymbol{J}_{\tilde{x}_{e} \nu_{e}} \boldsymbol{\nu}_{e, i n t}+\boldsymbol{J}_{\tilde{x}_{e} \nu_{e}} \boldsymbol{J}_{b} \boldsymbol{M}_{b}^{-1} \boldsymbol{A}^{T} \boldsymbol{h}, \\
& \dot{\boldsymbol{q}}=\boldsymbol{J}_{m}^{*-1} \boldsymbol{\nu}_{e, i n t},
\end{aligned}
$$

where the state is $\boldsymbol{z}=\left[\begin{array}{llll}\boldsymbol{h}^{T} & \boldsymbol{\nu}_{e, \text { int }}^{T} & \tilde{\boldsymbol{x}}_{e}^{T} & \boldsymbol{q}^{T}\end{array}\right]^{T} \in D$, with $D=\mathbb{R}^{18} \times Q$. Notice that the momentum is totally decoupled from the rest of the system and it has a linear first-order dynamics.

The stability of the closed-loop 21 is addressed in the following. Let us firstly define a region $\Omega$ which excludes the singularities of the generalized Jacobian $\boldsymbol{J}_{m}^{*}(\boldsymbol{q})$

$$
\Omega=\left\{\boldsymbol{z} \in D: \sigma_{\min }\left(\boldsymbol{J}_{m}^{*}(\boldsymbol{q})\right)>0\right\},
$$

where $\sigma_{\min }(\ll \cdot \gg)$ is the minimum singular value of the matrix $\ll \cdot \gg$. In the region $\Omega$ the Jacobian $\boldsymbol{J}_{m}^{*}$ is welldefined together with the dynamics matrices $M_{e}, C_{e}, C_{e h}$.

Proposition IV.1. The set of equilibria $z_{0}=\{\boldsymbol{z} \in \Omega: \boldsymbol{h}=$ $\left.\boldsymbol{\nu}_{e, \text { int }}=\tilde{\boldsymbol{x}}_{e}=\mathbf{0}\right\}$ is asymptotically stable.

Proof. $z_{0}$ is compact because $Q$ is compact. Then, the stability can be addressed in two steps using a cascade approach [12], showing first the asymptotic stability of the momentum 21a and then that of the robot subsystem 21b, 21c, 21d when $\boldsymbol{h}=\mathbf{0}$.

1) Since 21a is a linear first-order dynamics, it is asymptotic stable having chosen $\boldsymbol{D}_{h}$ positive definite.

2) In the subset $\boldsymbol{h}=\mathbf{0}$ the stability of 21b, 21c 21d can be addressed using the Lyapunov function

$$
V=\frac{1}{2} \boldsymbol{\nu}_{e, \text { int }}^{T} \boldsymbol{M}_{e} \boldsymbol{\nu}_{e, i n t}+\frac{1}{2} \tilde{\boldsymbol{x}}_{e}^{T} \boldsymbol{K}_{e} \tilde{\boldsymbol{x}}_{e}>0,
$$

which is always defined in $\Omega$. The time derivative along system trajectories is

$$
\begin{aligned}
& \dot{V}=\boldsymbol{\nu}_{e, i n t}^{T} \boldsymbol{M}_{e} \dot{\boldsymbol{\nu}}_{e, i n t}+\frac{1}{2} \boldsymbol{\nu}_{e, \text { int }}^{T} \dot{\boldsymbol{M}}_{e} \boldsymbol{\nu}_{e, i n t}+ \\
& +\boldsymbol{\nu}_{e, \text { int }}^{T} \boldsymbol{J}_{\tilde{x}_{e} \nu_{e}}^{T} \boldsymbol{K}_{e} \tilde{\boldsymbol{x}}_{e}=\frac{1}{2} \boldsymbol{\nu}_{e, \text { int }}^{T}\left(\dot{\boldsymbol{M}}_{e}-2 \boldsymbol{C}_{e}\right) \boldsymbol{\nu}_{e, \text { int }}+ \\
& \quad-\boldsymbol{\nu}_{e, \text { int }}^{T} \boldsymbol{D}_{e} \boldsymbol{\nu}_{e, \text { int }}=-\boldsymbol{\nu}_{e, \text { int }}^{T} \boldsymbol{D}_{e} \boldsymbol{\nu}_{e, i n t} \leq 0,
\end{aligned}
$$

where (14) has been used. Applying LaSalle $\tilde{\boldsymbol{x}}_{e} \rightarrow \mathbf{0}$ is obtained, thus proving the asymptotic stability.

From 1) and 2) then follows [12] the asymptotic stability of the closed-loop 21].

\section{A. Controller discussion}

It is relevant to analyze the final expression of the control actuation command, to remark the main advantage of the proposed internal/external decomposition and to gain further insight into the controller behavior.

The final expression of the control actuation command is obtained transforming back the controller given by (17), 20) through (12), as

$$
\begin{aligned}
\boldsymbol{f}_{b} & =-\boldsymbol{A}^{T} \boldsymbol{D}_{h} \boldsymbol{h}, \\
\boldsymbol{\tau} & =-\boldsymbol{J}_{m}^{* T}\left(\boldsymbol{J}_{\tilde{x}_{e} \nu_{e}}^{T} \boldsymbol{K}_{e} \tilde{\boldsymbol{x}}_{e}+\boldsymbol{D}_{e} \boldsymbol{\nu}_{e}\right)-\boldsymbol{M}_{b m} \boldsymbol{M}_{b}^{-1} \boldsymbol{A}^{T} \boldsymbol{D}_{h} \boldsymbol{h} .
\end{aligned}
$$

First, notice that the external actuator $f_{b}$ is only used to extract the momentum from the system and not to counterbalance any manipulator motion. This is the main advantage of the proposed $\boldsymbol{a}_{h}, \boldsymbol{f}_{e, \text { int }}$ decomposition and has a paramount importance for space robots, for which the external actuation is provided by nonrenewable energy sources like thrusters.

Second, notice that when the momentum is zero it follows $f_{b} \rightarrow \mathbf{0}$ and the proposed controller converges to the conventional free-floating generalized Jacobian control [1]:

$$
\boldsymbol{\tau}=-\boldsymbol{J}_{m}^{* T}\left(\boldsymbol{J}_{\tilde{x}_{e} \nu_{e}}^{T} \boldsymbol{K}_{e} \tilde{\boldsymbol{x}}_{e}+\boldsymbol{D}_{e} \boldsymbol{\nu}_{e}\right) .
$$

In conclusion, with the proposed controller all the operations will be performed in free-floating mode and the thrusters will be activated automatically only in case of external interactions, thus limiting the fuel consumption to the minimum needed. As a conclusive remark, notice the in the singularity of $\boldsymbol{J}_{m}^{*}$ the controller (25) does not fail computationally but an end effector task lying along a singular direction would result in zero torque and would not be executed [7].

\section{EXPERIMENTAL VALIDATION}

The proposed control methods are validated on the OOSSim (On-Orbit Servicing Simulator) HIL facility at DLR [13] (see Fig. 11). The OOS-SIM is a robotic simulator for space robots, which enables the testing of space manipulators on-ground before their actual deployment on-orbit. The test manipulator is mounted on a simulator manipulator in a micro-macro configuration. The simulator manipulator reproduces the satellite's dynamics based on a real-time model integration. The microgravity conditions in the test arm are replicated by actively compensating the joint gravity torques based on an identified model. The test manipulator is a KUKA KR4+ lightweight robot with seven degreesof-freedom. This arm is equipped with torque sensors and can be controlled both in position and torque. The simulator manipulator is a KUKA KR120 industrial robot, which is controlled in position and has no torque sensing.

The concept of the dynamics simulation is based on the integration of the first equation in (1) with the addition of a contact force $f_{e, e x t} \in \mathbb{R}^{6}$ on the end-effector:

$$
\boldsymbol{M}_{b} \dot{\boldsymbol{\nu}}_{b}=-\boldsymbol{M}_{b m} \ddot{\boldsymbol{q}}_{m s r}-\boldsymbol{C}_{b} \boldsymbol{\nu}_{b}-\boldsymbol{C}_{b m} \dot{\boldsymbol{q}}_{m s r}+\boldsymbol{f}_{b}+\boldsymbol{J}_{b}^{T} \boldsymbol{f}_{e, e x t},
$$


where $\dot{\boldsymbol{q}}_{m s r}$ and $\ddot{\boldsymbol{q}}_{m s r}$ are respectively the joint velocity and acceleration, obtained differentiating the measured joint angles $\boldsymbol{q}_{m s r}$ of the test arm. $\boldsymbol{f}_{e, e x t}$ can be measured using a $6 \mathrm{DOF}$ force-torque sensor on the $\mathrm{EE}$, or reconstructed using joint measurements of the test arm. In this paper, the second strategy has been used 5 . With this system, the dynamics can therefore be simulated taking into account real sensor noise, time delay, control discretization, model uncertainties and unmodelled dynamics of the test manipulator. On the other hand, the satellite dynamics is based on a model, whose parameters can be adapted depending on the specific scenario. In the present work, the satellite parameters used for the control and for the simulation are $m=150 \mathrm{~kg}$, $I_{x}=21.8 \mathrm{kgm}^{2}, I_{y}=15.0 \mathrm{kgm}^{2}, I_{z}=18.88 \mathrm{kgm}^{2}$. The robot parameters used for control have been obtained by fixed-base identification.

\section{A. Experimental results}

To show the effectiveness of the momentum dumping approach, experiments are conducted where the end effector is commanded in presence of an accumulated momentum. In the experiments, the momentum is induced in the system by an external contact on the end effector. The contacts are provided manually using a rod. The aim is to show that the end effector absolute pose is successfully regulated around a constant desired position, while the momentum is dumped from the system.

The gains used for the end effector stiffness are $k_{e, \text { trasl }}=$ $1000 \mathrm{~N} \mathrm{~m}^{-1}, k_{e, r o t}=70 \mathrm{~N} \mathrm{~m} \mathrm{rad}^{-1}$. The end effector damping gains have been designed using the method in [9]. For the momentum gains $d_{h, \text { trasl }}=20.28 \mathrm{~s}^{-1}, d_{h, \text { rot }}=$ $16 \mathrm{~m}^{2} \mathrm{~s}^{-1} \mathrm{rad}^{-1}$ are used. To show the need of a momentum dumping, we firstly analyze the system response in case of classical free-floating approach 26) and outline the main problem. After, we repeat the same experiment with the momentum-dumping control (25).

In Fig. 1. a snapshot sequence is shown for the freefloating robot in response to an accidental contact. It can be observed in Fig. 1 that the induced momentum leads to a drift of the whole system in the inertial space. The drift can be noticed also in Fig. 2, which shows that the base (yaw, pitch and roll) angles and the end effector position continue to grow after the contact. The end effector drift is related to the fact that the arm stretches in the attempt to hold the desired inertial position until a singularity is inevitably reached, compromising the end effector task.

To solve this problem, the impulse experiment is repeated with the proposed momentum dumping control. Fig. 3 shows the main results in terms of momentum, base attitude and end effector position. Fig. 3 shows that the momentum induced by the contact is successfully extracted from the system by the proposed momentum dumping control. Accordingly, the base drift is successfully stopped and the base pitch angle

\footnotetext{
${ }^{5}$ Notice that the external force is used here only as a mean to inject momentum into the system and is deactivated directly after the contact. No exact reconstruction is needed in the present work and the controller does not require any external force measurement.
}
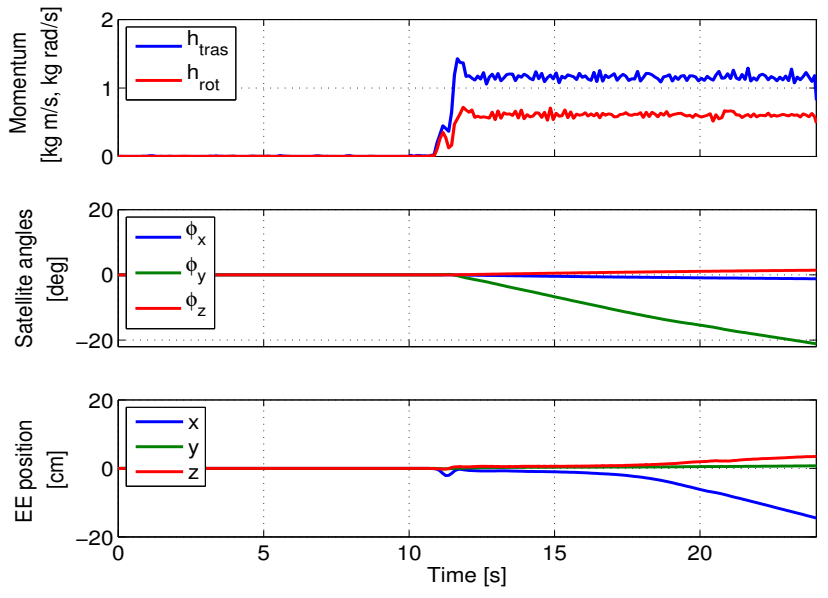

Fig. 2. Time response for free-floating control.
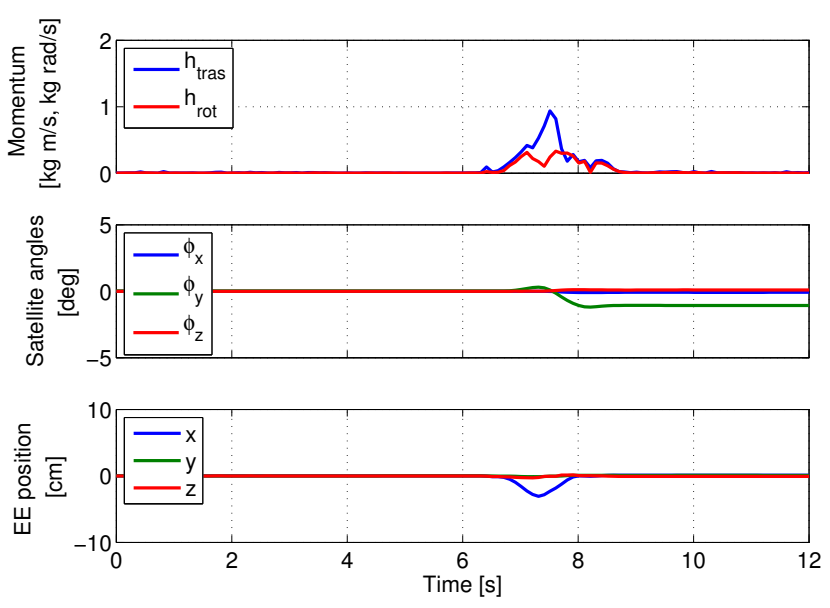

Fig. 3. Time response for momentum-dumping control.

converges to $\approx-1.5 \mathrm{deg}$. The end effector converges to the desired position with a precision of $1 \mathrm{~mm}$. This steadystate error is explained by the residual static friction in the joints, which acts as an external disturbance when the robot is commanded in torque mode. Finally, the satellite commanded forces and torques are shown in Fig. 4. The plot shows that the momentum-dumping is automatically activated as soon as the contact excites the momentum. The command signal is relatively noisy, due to the momentum computation. However, the noise is filtered out by the low-pass nature of the satellite and no relevant noise oscillations are visible in the base angles in Fig. 3. In conclusion, the experiments confirmed the effectiveness of the proposed control method.

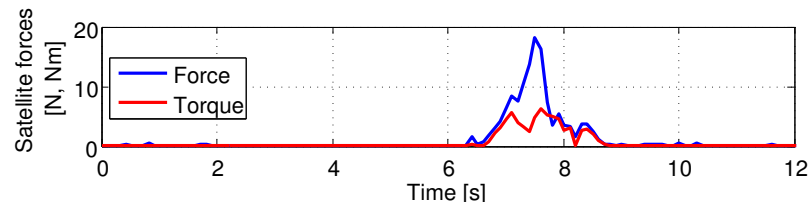

Fig. 4. Satellite commanded forces (norm) for momentum-dumping control. 


\section{B. Comments on the applicability}

The controller 25) requires feedback of $\tilde{\boldsymbol{x}}_{e}, \dot{\boldsymbol{q}}, \boldsymbol{h}$. In the experiment $\tilde{\boldsymbol{x}}_{e}$ is obtained from the forward kinematics of simulator and test arms. $\boldsymbol{h}$ is computed by (4), using the inertia model and a measurement of $\boldsymbol{R}_{\mathcal{T}, \mathcal{B}}$ and $\boldsymbol{\nu}_{b}$, extracting the former from the forward kinematics of the simulator arm and obtaining the latter by differentiation. In space, $\tilde{\boldsymbol{x}}_{e}$ could be measured by end effector cameras, the angular part of $\nu_{b}$ can be measured by gyros, while the linear part could be reconstructed by fusion of base-mounted cameras (or LIDARs) and accelerometers. Although the experiment showed good robustness to noise in the momentum, higher noise levels in the linear velocity are to be expected in space, since it is difficult to measure the linear velocity on orbit. An activation threshold for the dumping task $\boldsymbol{a}_{h}$ in 20 would be needed in the practice based on the noise levels of the velocity estimation.

Regarding the actuators, it has been assumed that the commanded base force $\boldsymbol{f}_{b} \in \mathbb{R}^{6}$ is actuated perfectly. However, in practice the minimum actuatable force would be limited by the minimum switching time of the thrusters' valves, thus the momentum could be dumped up to the actuator resolution. Due to the residual momentum, the base would then slowly drift in a free-floating fashion. Notice however that this residual drift would be notably less than the original accumulated momentum (e.g. due to an accidental impact), thus representing a valid improvement over a freefloating-only strategy. A possible remediation to this small drift could be to command the additional task of forcing the base to stay within a box in the inertial space or to absorb small momentum quantities into the wheels, if available.

\section{CONCLUSIONS AND FUTURE WORKS}

The inertial drift of a space robot in presence of accumulated momentum was addressed. Thanks to an internal/external motion decomposition, a Momentum Dumping Control has been derived to regulate a desired end-effector inertial pose while simultaneously extracting the momentum from the system. A feature of the proposed controller is that all robotic operations are performed in free-floating mode and the thrusters are activated automatically only in case of external interactions, thus limiting the fuel consumption to the minimum needed. The validity and applicability of the control method have been assessed with experiments.

Future works may further investigate the performance of the controller under noisy momentum measurements and thrust discretization effects.

\section{APPENDIX}

\section{MOMENTUM CONSTRAINT}

According to the Newton's third law, only external forces do change the total momentum of the system. Therefore, projecting the base external force $\boldsymbol{f}_{b}$ around $\mathcal{C}$, it must be

$$
\dot{\boldsymbol{h}}=\boldsymbol{A}^{-T} \boldsymbol{f}_{b}
$$

Let us now rederive $\dot{h}$ taking the time derivative of (4), as

$$
\dot{\boldsymbol{h}}=\dot{\boldsymbol{L}} \boldsymbol{v}+\boldsymbol{L} \dot{\boldsymbol{v}}=\left(\dot{\boldsymbol{L}}-\boldsymbol{L} \boldsymbol{M}^{-1} \boldsymbol{C}\right) \boldsymbol{v}+\boldsymbol{L} \boldsymbol{M}^{-1} \boldsymbol{f},
$$

where (1) was used. By algebraic manipulation it can be shown that $\boldsymbol{L} \boldsymbol{M}^{-1} \boldsymbol{f}=\boldsymbol{A}^{-T} \boldsymbol{f}_{b}$, thus (29) reduces to

$$
\dot{\boldsymbol{h}}=\left(\dot{\boldsymbol{L}}-\boldsymbol{L} \boldsymbol{M}^{-1} \boldsymbol{C}\right) \boldsymbol{v}+\boldsymbol{A}^{-T} \boldsymbol{f}_{b} .
$$

Comparing (28) and (30) for all $\boldsymbol{v} \in \mathbb{R}^{6+n}$ and $\boldsymbol{f}_{b} \in \mathbb{R}^{6}$, a constraint for the motion is then obtained as [10]

$$
\left(\dot{\boldsymbol{L}}-\boldsymbol{L} \boldsymbol{M}^{-1} \boldsymbol{C}\right) \boldsymbol{v}=\mathbf{0} .
$$

Thanks to the constraint (31) it can be conclusively shown that the Coriolis/centrifugal acceleration terms are identically zero for the momentum equation in $(13)$. This is obtained computing the first row of $\boldsymbol{C}$, which leads to:

$$
\left[\begin{array}{ll}
\boldsymbol{C}_{h} & -\boldsymbol{C}_{e h}^{T}
\end{array}\right]\left[\begin{array}{c}
\boldsymbol{h} \\
\boldsymbol{\nu}_{e, i n t}
\end{array}\right]=-\boldsymbol{M}_{c}^{-1}\left(\dot{\boldsymbol{L}}-\boldsymbol{L} \boldsymbol{M}^{-1} \boldsymbol{C}\right) \boldsymbol{v}=\mathbf{0},
$$

where (31) has been applied.

\section{REFERENCES}

[1] Y. Masutani, F. Miyazaki, and S. Arimoto, "Sensory feedback control for space manipulators," in Robotics and Automation, IEEE International Conference on, May 1989, pp. 1346-1351 vol.3.

[2] K. Nanos and E. Papadopoulos, "On the use of free-floating space robots in the presence of angular momentum," Intelligent Service Robotics, vol. 4, no. 1, pp. 3-15, 2011.

[3] D. N. Nenchev and K. Yoshida, "Impact analysis and post-impact motion control issues of a free-floating space robot subject to a force impulse," IEEE Transactions on Robotics and Automation, vol. 15, no. 3, pp. 548-557, Jun 1999.

[4] S. Xu, H. Wang, D. Zhang, and B. Yang, "Adaptive zero reaction motion control for free-floating space manipulators," IEEE Transactions on Aerospace and Electronic Systems, vol. 52, no. 3, June 2016.

[5] A. M. Giordano, G. Garofalo, M. De Stefano, C. Ott, and A. AlbuSchäffer, "Dynamics and control of a free-floating space robot in presence of nonzero linear and angular momenta," in Proceedings of the 55th IEEE Conference on Decision and Control, Dec 2016.

[6] H. Nakanishi and K. Yoshida, "Impedance Control for Free-flying Space Robots -Basic Equations and Applications-," in Intelligent Robots and Systems, 2006 IEEE/RSJ International Conference on, Oct 2006.

[7] S. Dubowsky and E. Papadopoulos, "The kinematics, dynamics, and control of free-flying and free-floating space robotic systems," Robotics and Automation, IEEE Transactions on, Oct 1993.

[8] G. Garofalo, C. Ott, and A. Albu-Schäffer, "On the closed form computation of the dynamic matrices and their differentiations," in Intelligent Robots and Systems (IROS), 2013 IEEE/RSJ International Conference on, Nov 2013, pp. 2364-2359.

[9] C. Ott, Cartesian impedance control of redundant and flexible-joint robots. Springer, 2008.

[10] G. Garofalo, B. Henze, J. Englsberger, and C. Ott, "On the inertially decoupled structure of the floating base robot dynamics," IFACPapersOnLine, vol. 48, no. 1, pp. 322 - 327, 2015, 8th Vienna International Conferenceon Mathematical ModellingMATHMOD 2015.

[11] O. Khatib, "A unified approach for motion and force control of robot manipulators: The operational space formulation," Robotics and Automation, IEEE Journal of, vol. 3, no. 1, pp. 43-53, February 1987.

[12] M. I. El-Hawwary and M. Maggiore, "Reduction theorems for stability of closed sets with application to backstepping control design," Automatica, vol. 49, no. 1, pp. 214 - 222, 2013.

[13] J. Artigas, M. D. Stefano, W. Rackl, R. Lampariello, B. Brunner, W. Bertleff, R. Burger, O. Porges, A. Giordano, C. Borst, and A. AlbuSchaeffer, "The OOS-SIM: An on-ground simulation facility for on-orbit servicing robotic operations," in 2015 IEEE International Conference on Robotics and Automation (ICRA), May 2015. 